\title{
Borderline Serous Cystadenoma Presenting as Umbilical Mass Post Hysterectomy and Bilateral Salpingo Ooporectomy: A Case Report and Literature Review
}

\author{
Elizabeth Ande, Peter Hinstridge, Beena Subba*, Anupriya Gupta, Deepak Kumar, Shradha Limbu
}

Department of Obstetrics \& Gynaecology, North Middlesex University, London, UK

"Corresponding author: Beena Subba, Department of Obstetrics \& Gynaecology, North Middlesex University, Teaching Hospital, Sterling Way, Edmonton, N181QX, London, United Kingdom. Tel: +44-7823847423; Email: b.subba@nhs.net

Citation: Ande E, Hinstridge P, Subba B, Gupta A, Kumar D, et al. (2019) Borderline Serous Cystadenoma Presenting as Umbilical Mass Post Hysterectomy and Bilateral Salpingo Ooporectomy: A Case Report and Literature Review. Arch Surg Clin Case Rep 02: 114. DOI: $10.29011 /$ ASCR-114/100114

Received date: 06 August, 2019; Accepted date: 13 August, 2019; Published Date: 20 August, 2019

\section{Introduction}

One of the initial description of Serous Borderline Ovarian Tumour (BOT) dates back to 1929 by Taylor [1]. Borderline Ovarian Tumours (BOT) are basically abnormal cells, noninvasive in nature that are formed in the epithelium of the ovary. They account for $15-20 \%$ of ovarian tumours [2,3]. A major fraction of the BOT is of Serous variety followed by Mucinous and then other less common histological variants. They typically have excellent prognosis with 10-year survival being nearly $94 \% .2$

Umbilical masses presenting as BOT are a relatively rare entity.4Although these are estimated to occur in less than $0.1 \%$ of Tumour masses it should be considered as part of the content of an umbilical mass [4]. BOT usually occur in young women with a favorable prognosis with an incidence of 1.8-4.8/100000 [2]. The recent rise in the incidence of BOT has been attributed to the advancement in pathological diagnosis.

The authors present a case report of a woman who had borderline serous cystadenoma presenting as an umbilical mass post Laparoscopically Assisted Vaginal Hysterectomy (LAVH) with Bilateral Salpingo Oophorectomy (BSO). This case report emphasizes the need to consider other etiologies of umbilical masses and not merely attribute them to hernias.

\section{Case report}

A 63-year-old para four lady with background of TIA, hypertension and diabetes, presented to the general surgery outpatient clinic at the authors' institution. She had a history of recurrent infraumbilical mass since the age of 56. On examination the mass was $8 \mathrm{~cm} \times 6 \mathrm{~cm}$, non-tender, cystic and solid in nature, multiloculated and fixed in lower aspects. A provisional diagnosis of asymptomatic recurrent reducible hernia was made and she had a CT scan that demonstrated a soft tissue subcutaneous mass which was $3.8 \mathrm{~cm} \mathrm{x} 7.6 \mathrm{~cm}$ to the left of the umbilicus. She underwent an excisional biopsy with histopathological finding of a borderline serous cyst adenoma and is currently being followed up at the outpatient clinic of the referral tertiary hospital.

She presented with Post-Menopausal Bleeding (PMB) at age 53, endometrial biopsy and polypectomy revealed a benign polyp with secretory endometrium on histology. When she was 59 years old, she had a polyp removed for recurrent PMB that revealed a complex hyperplasia. She subsequently underwent LAVH with BSO and the histology confirmed atypical hyperplasia of the endometrium while the ovaries showed prominent surface stromal proliferation with numerous surface epithelial invaginations and surface epithelial inclusion cysts with Psammomatous calcification. Similar cysts were also present on the serosal surface of the uterus. No definite atypia or malignancy was seen.

\section{Discussion}

Serous BOT have been associated with peritoneal implants where there is increased epithelial proliferation, nuclear atypia but no stromal invasion. This is in contrast to epithelial ovarian cancer. Serous epithelial ovarian carcinoma (most common epithelial ovarian cancer type) is usually diagnosed in the elderly, associated with metastasis and poor prognosis. BOT are rarely seen in BRCA and K-Ras mutations but the epidemiological risk factors for BOT are similar to the risk factors for ovarian carcinoma [2]. BOT is associated with a risk of recurrence of $11 \%$ and transformation to malignancy of 20 to $30 \%$ [2]. Despite the good prognosis associated with BOT, some patients can develop the aggressive form which can lead to morbidity and mortality. 


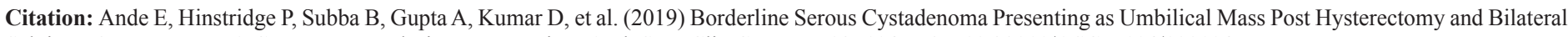
Salpingo Ooporectomy: A Case Report and Literature Review. Arch Surg Clin Case Rep 02: 114. DOI: 10.29011/ASCR-114/100114

The management of BOT in young women with uncompleted family size can be quite challenging, and even though the patient in the present study had completed her family size, her management will also pose a challenge as the umbilical mass (a noninvasive implant) is associated with recurrence. It is uncommon to have Serous cystadenoma presenting as an umbilical mass. The classical Sister Josephs Nodule (SJN) is a representation of the progression of a malignancy and could be a result of lymphatic, haematogenous or embryonic ligaments spread [5]. The presence of a palpable periumbilical nodule could be the first sign of an underlying malignancy [6] which could be gastrointestinal, extra abdominal (lungs or breast) or genital tract and is usually associated with a poor prognosis [5]. In female patients consideration of a gynecological malignancy should usually be the first step, in our case she had undergone hysterectomy and BSO. In this patient however it is pertinent to note that the umbilical mass was not a malignancy.

Nonspecific symptoms are the most common presentation of BOT, followed by lack of symptoms and then bleeding abnormalities [2]. She however, presented to us with a recurrent umbilical mass. The occurrence of a strangulated and incarcerated hernia in a prior case report which was confirmed histologically to be a Serous carcinoma has also been described in a 54-year-old lady leading to hysterectomy and BSO with identification of the left ovarian serous carcinoma after the surgery [5]. The application of imaging to accurately characterize the mass is extremely important as it aids biopsy and prompt decision making [6].

The imaging modalities available to help in delineating the nature of the mass are ultrasound scan (which is quite accurate when performed by an experienced operator) and CT scan $[2,6]$. Therefore, ultrasound scan complemented by CT scan should be the imaging modality to guide biopsy and further management of atypical umbilical masses. Mucinous Cyst Adenoma of the appendix and mucinous cystadenoma with disseminated peritoneal adenomucinous have been reported in patients with reducible mass of umbilical site. They occur rarely but develop as a consequence of proliferation of mucin secreting cells $[7,8]$. Port site metastasis following laparoscopic surgery for BOT mucinous tumor without spillage has also been reported and the laparoscopic route for cystectomy can result in cyst rupture and incomplete staging [9].

The Serous types of BOT are further divided into typical Serous BOT or Micro papillary type which is more commonly associated with recurrence [2]. The occurrence of extra ovarian spread in Serous borderline tumours even though non aggressive and associated with a good prognosis, when of the micro papillary pattern can be associated with recurrence $[3,10]$. This was the case where a 70-year lady following hysterectomy and bilateral oophorectomy developed 6 recurrences of Serous micro papillary tumour in various parts of her body over a 30-year span [10]. This emphasizes the need for long term follow up for patients with serous borderline tumour. A similar case has been reported where a 75-year-old lady had surgery for umbilical hernia which turned out to be a recurrent borderline papillary serous cystadenoma, she had also had hysterectomy with ovarian conservation years earlier, similar to our patient who had a hysterectomy and BSO [11].

\section{Conclusion}

This case report contradicts the notion that all umbilical masses are hernias and nullifies the common misconception. It also alerts us to be wary of umbilical masses as purely surgical in nature. Literature reports a substantial number of cases with umbilical mass diagnosed as malignant with the ovary being the primary tumour or umbilical masses following a past TAH and BSO for a known ovarian malignancy. The case presented here to the best of our knowledge is the first case report where a Serous cystadenoma presented as an umbilical mass years following LAVH, BSO, where the ovarian histology had revealed inclusion cysts and stromal proliferation. As the prognosis is good even for extra ovarian spread she will be followed up at the outpatient clinic.

\section{References}

1. Taylor HC (1929) Malignant and semi malignant tumours of the ovary. Surg Gynecol Obstet 48: 204-230.

2. Fischerova D, Zikan M, Dundr P, Dibula D (2012) Diagnosis, treatment and follow up of borderline ovarian tumours. The oncologist 17: 1515-1533.

3. Faten L, Mouna M (2019) Ovarian cystadenoma. StatPearls (Internet). Treasure Island(FL): Stat Pearls Publishing.

4. Arumugham S, Mathew M, Al Kalbani M, Al Wahaibi KN (2015) Incarcerated umbilical hernia as an initial presentation of advanced ovarian malignancy. Med J Obstet Gynaecol 3: 1062.

5. Sahim S, Borekci H, Akyuz Y, Erkoc M, Karacabey S, et al. (2015) Metastatic serous carcinoma initially presented as an incarcerated and strangulated umbilical hernia: a rare case report. American Journal of cancer case reports 3 .

6. Gibbs TS (2012) Sister Mary Joseph Nodule: A cohort study of an unusual umbilical mass. Journal of diagnostic medical sonography 28 : 107-117.

7. Singh VK, Nishant K, Sharma BK, Lamichaney R (2014) A weeping umbilical hernia:bilateral ovarian mucinous cystadenoma with disseminated peritoneal adenomucinosis. BMJ Case Rep.

8. Ren B, Meng X, Cao Z, Guo C, Zhang Z (2016) Mucinous cystadenoma of the appendix presenting as an umbilical hernia: A case report. Oncology letters 11: 4200-4202.

9. Furukawa N, Nishioka K, Noguchi T, Kajihara H, Horie K (2014) Port site metastasis of mucinous borderline ovarian tumour after laparoscopy. Case Rep 'oncol 7: 804-809. 
Citation: Ande E, Hinstridge P, Subba B, Gupta A, Kumar D, et al. (2019) Borderline Serous Cystadenoma Presenting as Umbilical Mass Post Hysterectomy and Bilateral Salpingo Ooporectomy: A Case Report and Literature Review. Arch Surg Clin Case Rep 02: 114. DOI: 10.29011/ASCR-114/100114

10. Minagawa M, Maeda M, Shimauchi M, Kishi H, Teshima S (2018) Serous Bordeline tumour with micropapillary pattern of the right ovary that developed 6 recurrences over 30 years after primary surgery. Gynecol Oncol Rep 25: 45-47.
11. Williams T (2006) Recurrence of borderline papillary serous tumours: a modern dilemma. Gynecol Surg 3: 49-51. 